or since ${ }^{n a+\beta} P_{a+\gamma}$ gives the unrestricted number of ways of filling up the places, the probability that no object is in a place correspondingly marked in a fortuitous distribution is

$$
\sum_{k=0}^{a} \frac{(-n)^{k}}{\underline{L}} \cdot \frac{{ }^{a} C_{k}}{n a+\beta} C_{k} .
$$

The particular result for the problem enunciated at the beginning is got by putting $n=1, \beta=\gamma=0$

$$
\text { i.e. } \sum_{i=0}^{a} \frac{(-1)^{k}}{\underline{\underline{k}}}
$$

or $\quad$ a $\sum_{k=0}^{a} \frac{(-1)^{k}}{\underline{\mid k}}$ according to the mode of statement of the problem.

William Milier.

\title{
Analytical Note on Lines Forming a Harmonic Pencil.
}

The following is a simple proof of the theorem that the concurrent lines whose equations are

$$
\begin{aligned}
& a_{1} x+b_{1} y+c_{1}=0 \\
& a_{2} x+b_{3} y+c_{3}=0 \\
& a_{1} x+b_{1} y+c_{1}=k\left(a_{2} x+b_{2} y+c_{2}\right) \text {. } \\
& a_{1} x+b_{1} y+c_{1}=-k\left(a_{2} x+b_{2} y+c_{2}\right)
\end{aligned}
$$

form a harmonic pencil.

Let a line through the origin parallel to the line (2) intersect (4) in $A\left(x_{1}, y_{1}\right)$, and (3) in $B\left(x_{2}, y_{2}\right)$.

The pencil is harmonic if the mid-point of $A B, C\left(\frac{x_{1}+x_{2}}{2}, \frac{y_{1}+y_{2}}{2}\right)$, lies on (1).

Since $O A B$ is parallel to (2) we have

$$
a_{2} x_{1}+b_{2} y_{1}=a_{2} x_{2}+b_{2} y_{2}=0 \text {. }
$$

Hence since $A\left(x_{1}, y_{1}\right)$ lies on (4),

$$
a_{1} x_{1}+b_{1} y_{1}+c_{1}=-k c_{2},
$$


and since $B\left(x_{2}, y_{2}\right)$ lies on (3)

$$
a_{1} x_{2}+b_{1} y_{2}+c_{1}=+k c_{2} \text {. }
$$

Adding, and dividing by 2,

$$
a_{1} \frac{x_{1}+x_{2}}{2}+b_{1} \frac{y_{1}+y_{2}}{2}+c_{1}=0 .
$$

Therefore the mid-point of $A B$ lies on (1).

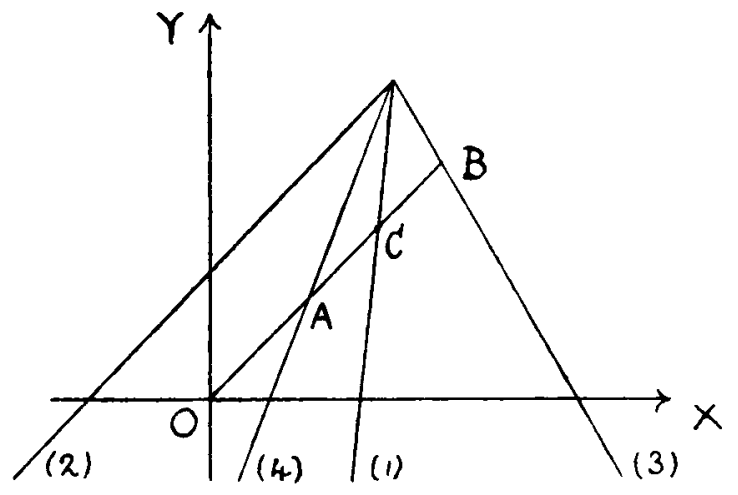

N. M'Arthur.

\section{Trigonometrical Ratios of the half-angles of a Triangle (Geometrical Proofs).}

1. $A B C$ is a triangle; bisect angle $A$ by $A E$; produce $A B$; draw $B D F$ and $C E G$ perpendicular to $A E$; join $F G$.

$G C F B$ is a cyclic trapezium

$$
\begin{aligned}
& \therefore G C . F B+C F . B G=B C . F G \text {, } \\
& \therefore \quad 2 E C .2 D B+(b-c)^{2}=a^{2} \text {, } \\
& \therefore \quad 4 E C, D B=a^{2}-(b-c)^{2} \\
& =(a-b+c)(a+b-c) \\
& =4(s-b)(s-c) \text {, } \\
& \therefore \quad E C, D B=(s-b)(s-c) \text {. }
\end{aligned}
$$

